\title{
Rosacea and Chronic Rhinosinusitis: A Case-Controlled Study
}

\author{
Amal O. Al-Balbeesi \\ Department of Dermatology (82), King Khalid University Hospital, King Saud University, Riyadh, Saudi Arabia
}

\section{Key Words}

Rosacea · Chronic rhinosinusitis · Cathelicidin

\begin{abstract}
Objective: To determine the relationship between rosacea, chronic rhinosinusitis (CRS), and the clinical presentation of rosacea. Subjects and Method: Twenty-eight female Saudi patients diagnosed with rosacea at the Dermatology Clinic, King Khalid University Hospital, King Saud University, Riyadh, Saudi Arabia, between September 2011 and September 2012 and 20 age- and sex-matched control patients were included in the study. Paranasal sinus X-rays and assessments of the serum concentration of IgE (ImmunoCAP test; Phadia Laboratory Systems) were performed in both groups. Result: The rosacea patients had significantly more radiological evidence of CRS than the patients without rosacea [19 (67.9\%) vs. 4 (20\%), $p=0.003]$. The median IgE concentration was similar in both groups (225.4 vs. $223.1 \mathrm{kU} / \mathrm{l}$ ). Nine rosacea patients $(32.1 \%)$ without radiological evidence of CRS did not have a significantly different median concentration of IgE compared with those who had radiological evidence of CRS (190.5 vs. $111.5 \mathrm{kU} / \mathrm{l}, \mathrm{p}=0.859$ ). Erythematotelangiectatic severity was significantly associated with CRS ( $p$ $=0.038$ ). Serum IgE did not correlate with the severity of the
\end{abstract}

\begin{tabular}{ll}
\hline KARGER & $\begin{array}{l}\text { C 2014 S. Karger AG, Basel } \\
\text { 1011-7571/14/0236-0511\$39.50/0 Open cer }\end{array}$ \\
E-Mail karger@karger.com & $\begin{array}{l}\text { This is an Open Access article licensed under the terms of the } \\
\text { www.karger.com/mpp }\end{array}$ \\
& $\begin{array}{l}\text { Creative Commons Attribution-NonCommercial 3.0 Un- } \\
\text { ported license (CC BY-NC) (www.karger.com/OA-license), } \\
\text { applicable to the online version of the article only. Distribu- } \\
\text { tion permitted for non-commercial purposes only. }\end{array}$
\end{tabular}

facial condition. Conclusion: Patients with rosacea and CRS manifested severe erythematotelangiectatic rosacea. There was enough evidence to suggest an association between rosacea and CRS. Clinical and radiological assessments of the paranasal sinuses are recommended.

C 2014 S. Karger AG, Basel

\section{Introduction}

Rosacea is a common disease, especially in fairskinned individuals; however, any ethnic group may be affected [1]. Molecular studies have shown that an altered innate immune response is involved in the pathogenesis of the vascular and inflammatory disease seen in patients with rosacea [2]. Environmental changes, altered hormone balances, ultraviolet light, and microbes are sensed by Toll-like receptors and other pattern recognition receptors. Toll-like receptor signaling induces effector molecules such as cathelicidin, matrix metalloproteinases, reactive oxygen species, nitric oxides, cytokines, and chemokines $[3,4]$. These effectors modify the dermal structure by vascular changes and collagen degeneration associated with inflammatory cell recruitment [5-7].
Dr. Amal O. Al-Balbeesi, MD

Department of Dermatology (82)

King Khalid University Hospital, King Saud University

PO Box 2925, Riyadh 11461 (Saudi Arabia)

E-Mail amalbalbeesi@gmail.com 
Cathelicidin is an antimicrobial peptide that is abundant in rosacea patients. Cathelicidin promotes and regulates leukocyte chemotaxis, angiogenesis, and the expression of extracellular matrix components. The combination of these creates the irreversible vascular changes seen in rosacea $[2,6]$.

Cathelicidin expression has been identified in many cells and tissues including T cells, B cells, NK cells, macrophages, eosinophils, Langerhans cells, mast cells, CD1aexpressing dendritic cells, gastric cells, airway epithelial cells, and nasal mucosal epithelium [8-10]. Ooi et al. [11] reported an increased expression of cathelicidin in nasal mucosal biopsies of patients with chronic rhinosinusitis (CRS) in response to the stimulation of innate immunity. This overexpression of cathelicidin that had been reported to occur in rosacea and CRS formulated the rationale for our study.

\section{Subjects and Methods}

A total of 1,321 patients were prospectively screened for rosacea at the Dermatology Clinic of King Khalid University Hospital, King Saud University, Riyadh, Saudi Arabia, between September 2011 and September 2012. The diagnosis of rosacea included findings of midfacial erythema, telangiectasia, papules, and pustules. The Burton Wave Plus Fluorescent Magnifier Light (3.5 dpt) was used to visualize the erythema and telangiectasia in skin types 5 and 6. High-resolution digital photographs were taken using Canfield VISIA Complexion Analysis (Fairfield, N.J., USA) and analyzed for facial redness and telangiectasia in type 6 skin [12]. The study group consisted of 28 patients with clinically diagnosed rosacea, and the control group included 20 patients with dermatological diseases other than rosacea. The study group patients were divided into two subgroups based on the presence or absence of radiological findings of CRS. The Institutional Review Board of the College of Medicine, King Saud University, approved the study. Written informed consent was obtained from all participants in both groups.

Information collected as part of a detailed history of rosacea included the following: duration; aggravating factors; family history; cutaneous symptoms including scaling, pruritus, and skin tightness; presence and duration of any atopic disease (allergic rhinitis, allergic conjunctivitis, and bronchial asthma); symptoms and signs of CRS (postnasal drip, facial tenderness, anosmia, and headache), and migraine (because of its possible association with rosacea). Patients with Helicobacter pylori infection were excluded from the study due to the possible pathogenic role of $H$. pylori in rosacea [13]. The Fitzpatrick skin phototype system [14] was used to classify skin type: light brown is specified as type 4 , medium brown as type 5 , and dark brown as type 6 .

A grading system from the National Rosacea Society Expert Committee [15] was used to assess the subtypes of the disease. Subtype 1, erythematotelangiectatic rosacea (ETR; characterized by episodic flushing or persistent facial erythema and telangiectasia), was rated as follows: absence of cutaneous signs $=0$; mild signs $=$
Table 1. Comparison of rosacea patients and control patients

\begin{tabular}{lccl}
\hline & $\begin{array}{l}\text { Rosacea patients } \\
(\mathrm{n}=28)\end{array}$ & $\begin{array}{l}\text { Control patients } \\
(\mathrm{n}=20)\end{array}$ & $\begin{array}{l}\mathrm{p} \\
\text { value }\end{array}$ \\
\hline Age, years & $41.6 \pm 5.9$ & $37.9 \pm 8.4$ & 0.091 \\
IgE level, kU/l & $223.9 \pm 244.8$ & $223.12 \pm 254$ & 0.252 \\
Median IgE level, kU/1 & 225.4 & 223.1 & 0.2186 \\
$\begin{array}{l}\text { Patients with high IgE } \\
\text { Atopic diseases }\end{array}$ & $13(46.4)$ & $5(25.0)$ & \\
$\quad$ & & & \\
$\quad$ Yes & $21(75.0)$ & $12(60)$ & 0.193 \\
$\quad$ No & $7(25.0)$ & $8(40)$ & \\
$\begin{array}{l}\text { X-ray findings } \\
\text { Yes }\end{array}$ & $19(67.9)$ & $4(20)$ & 0.003 \\
$\quad$ No & $9(32.1)$ & $16(80)$ & \\
\hline
\end{tabular}

Values are mean $\pm \mathrm{SD}$ or $\mathrm{n}(\%)$.

$1 ;$ moderate signs $=2$, and severe signs $=3$. Subtype 2 , papulopustular rosacea (PPR; characterized by persistent facial erythema with transient papules, pustules, or both), was rated as follows: absence of cutaneous signs $=0$; mild signs $=1 ;$ moderate signs $=2$, and severe signs $=3$. The control group included patients with any skin condition other than rosacea, such as telogen effluvium, androgenic alopecia, psoriasis, urticaria, vitiligo, melasma, and discoid lupus. The patients in the control group were asked about the presence of symptoms and signs of CRS or any atopic disorders. Paranasal sinus X-rays (Caldwell's, Waters', and lateral views) were performed in both groups. Fluid level, mucosal thickening, dense shadows, and haziness in maxillary, frontal, or ethmoid sinuses were diagnostic of sinusitis [16]. A paranasal sinus X-ray, rather than CT scanning, was performed as a preliminary interventional investigation, which was sufficient enough to yield a positive or a negative diagnosis of CRS because paranasal sinus X-ray is the standard image for diagnosing chronic sinusitis due to its lower radiation level, lower costs, patient safety, and the acceptable level of accuracy of $54 \%$ (vs. $68 \%$ for CT) [17]. However, paranasal sinus CT scanning has been recommended and is considered the gold standard for diagnosing recurrent CRS, providing preoperative anatomical details that justify the higher radiation level [18].

An assessment of the serum IgE concentration (ImmunoCAP test; Phadia Laboratory Systems), which supports the diagnosis of atopic diseases, was done in all patients [19]. A level above $100 \mathrm{kU} / \mathrm{l}$ was considered significant. Data were collected and analyzed using SPSS software version 18 . The group statistics were assessed using Student's t test for paired samples and Pearson's correlation test to determine the correlation between variables.

\section{Results}

Of the 28 rosacea patients, $10(35.7 \%)$ had skin type 4 , 3 (10.7\%) had skin type 5 , and $15(53.6 \%)$ had skin type 6. The mean age for patients with rosacea was $41.6 \pm 5.9$ years compared with $37.9 \pm 8.4$ years in the control group. 
Table 2. Comparison between rosacea patients with radiological evidence of sinusitis and rosacea patients without sinusitis

\begin{tabular}{|c|c|c|c|}
\hline & \multicolumn{2}{|c|}{$\mathrm{X}$-ray evidence of sinusitis } & \multirow[t]{2}{*}{$\mathrm{p}$ value } \\
\hline & yes $(n=19)$ & no $(\mathrm{n}=9)$ & \\
\hline Age, years & $41.0 \pm 5.9$ & $42.8 \pm 6.3$ & 0.471 \\
\hline Duration, years & $5.0 \pm 3.9$ & $4.3 \pm 6.2$ & 0.715 \\
\hline IgE concentration, $\mathrm{kU} / \mathrm{l}$ & $214.4 \pm 274.7$ & $241.4 \pm 195.9$ & 0.821 \\
\hline Median IgE concentration, kU/l & 111.50 & 190.50 & 0.8586 \\
\hline \multicolumn{4}{|l|}{ Skin type } \\
\hline 4 & $7(36.8)$ & $3(33.3)$ & \multirow{3}{*}{0.356} \\
\hline 5 & $3(15.8)$ & $0(0.0)$ & \\
\hline 6 & $9(47.4)$ & $6(66.7)$ & \\
\hline \multicolumn{4}{|l|}{ Erythematotelangiectatic severity } \\
\hline 1 & $0(0.0)$ & $0(0.0)$ & \multirow{3}{*}{0.0038} \\
\hline 2 & $8(42.1)$ & $6(66.7)$ & \\
\hline 3 & $11(57.9)$ & $3(33.3)$ & \\
\hline \multicolumn{4}{|l|}{ Papulopustular severity } \\
\hline 1 & $0(0.0)$ & $0(0.0)$ & \multirow{3}{*}{0.329} \\
\hline 2 & $13(68.4)$ & $4(44.4)$ & \\
\hline 3 & $3(15.8)$ & $1(11.1)$ & \\
\hline \multicolumn{4}{|l|}{ Atopic diseases } \\
\hline Yes & $14(73.7)$ & $7(77.8)$ & \multirow[t]{2}{*}{0.602} \\
\hline No & $5(26.3)$ & $2(22.2)$ & \\
\hline \multicolumn{4}{|l|}{ Migraine } \\
\hline Yes & $3(15.8)$ & $0(0.0)$ & \multirow[t]{2}{*}{0.207} \\
\hline No & $16(84.2)$ & $9(100)$ & \\
\hline \multicolumn{4}{|l|}{ Family history } \\
\hline Yes & $2(10.5)$ & $1(11.1)$ & \multirow[t]{2}{*}{0.139} \\
\hline No & $19(100)$ & $8(88.9)$ & \\
\hline \multicolumn{4}{|l|}{ Cutaneous symptoms } \\
\hline Scaling & $19(100)$ & $9(100)$ & \\
\hline Pruritus & $19(100)$ & $9(100)$ & \\
\hline Skin tightness & $19(100)$ & $9(100)$ & \\
\hline Sinusitis symptoms/signs & $13(68.4)$ & $3(33.3)$ & \\
\hline \multicolumn{4}{|l|}{ Aggravating factors } \\
\hline Sun & $19(100)$ & $9(100)$ & \\
\hline Heat & $8(47.4)$ & $2(22.2)$ & $<0.0001$ \\
\hline Dust & $5(26.3)$ & $1(11.1)$ & $<0.0001$ \\
\hline Sweat & $3(15.8)$ & $0(0.0)$ & $<0.0001$ \\
\hline
\end{tabular}

Values are mean \pm SD or $\mathrm{n}(\%)$.
CRS in Rosacea Patients versus the Control Group

Of the 28 rosacea patients, $19(67.9 \%)$ had radiological evidence of CRS, while 4 patients (20\%) in the control group had CRS ( $\mathrm{p}=0.003$, OR $=2.884$; 95\% CI: 1.179 7.051). The median IgE concentrations were $225.4 \mathrm{kU} / \mathrm{l}$ in the study group and $223.1 \mathrm{kU} / \mathrm{l}$ in the control group $(\mathrm{p}=0.219$; table 1$)$.

\section{Clinical Features and IgE Concentration in the \\ Rosacea Subgroups}

Patients with rosacea and CRS had more severe ETR than rosacea patients without CRS [11 (57.9\%) vs. $3(33.3 \%), p=0.0038$; table 2]. ETR severity was also significantly correlated with the presence of atopic diseases (bronchial asthma and allergic rhinitis with or without allergic conjunctivitis; $\mathrm{r}=6.30, \mathrm{p}=0.012$ ).

No significant difference in the median IgE concentration was observed between rosacea patients with and those without radiological evidence of CRS (111.50 vs. $190.5 \mathrm{kU} / \mathrm{l}, \mathrm{p}=0.859$; table 2). Comparative analyses of the subgroups of rosacea patients showed no significant distinction in skin type, PPR severity, the presence of atopic diseases, and migraine ( $\mathrm{p}>0.05$; table 2 ). In both subgroups, the duration of the disease was not significantly correlated with ETR severity $(\mathrm{r}=10.8, \mathrm{p}=0.373)$ and PPR severity $(r=30.03, p=0.464)$. None of the patients presented with the granulomatous variant of rosacea during the study period. 
Sun exposure (reported by 28 patients, $100 \%$ ) was the most common aggravating factor of rosacea in both subgroups, while heat was reported by $8(47.4 \%)$, dust by 5 (26.3\%), and sweat by 3 patients (15.8\%) with radiological evidence of CRS, reaching statistical significance for all comparisons $(\mathrm{p}<0.0001)$ when compared with the patients without radiological evidence of CRS. Heat, dust, and sweat were reported by 2 (22.2\%), 1 (11.1\%), and 0 $(0 \%)$ of the patients without radiological evidence of CRS, respectively.

\section{Discussion}

This study showed that patients with rosacea were significantly more likely to have radiological evidence of CRS, thereby indicating an association between rosacea and CRS. Patients with rosacea and radiological evidence of CRS had more severe ETR than those without radiological evidence of CRS. It seems possible to say that there is a tissue interaction between the skin and the nasal mucosa mediated by cathelicidin, anatomical proximity, and vascular unity. ETR severity was also significantly correlated with atopic diseases but not with $\operatorname{IgE}$ level. Secondary to the high prevalence of atopic diseases among all patients of the study, statistical analysis showed that the differences in median IgE levels between the rosacea patients and the control group and between the rosacea patients with and those without $(190.5 \mathrm{kU} / \mathrm{l})$ radiological evidence of CRS were not significant ( $p>0.05$ for all comparisons). These findings confirmed those of a previous study by Dirschka et al. [19], who reported that high IgE levels did not show any statistical significance between rosacea patients and control patients. On the other hand, the authors reported that perioral dermatitis showed a statistically significant relationship to IgE levels $\geq 100 \mathrm{kU} / \mathrm{l}$ and atopic diseases if compared between control and rosacea patients ( $\mathrm{p}<0.001$ for all comparisons). It seems plausible to suggest that there might be a subset of rosacea that simulates perioral dermatitis by exhibiting a higher incidence of atopic diseases and high levels of IgE.

The percentages of the patients who presented with the ETR subtype (100\%) and of those who presented with the PPR subtype (71.4\%) were higher than the 81 and 19\%, respectively, reported by Berg and Lidén [20] for the two subtypes. Possible explanations for the higher incidence rates in our population for both subtypes of rosacea as compared with those of Berg and Lidén are the influence of the underlying CRS or their exclusion of telangiectasia

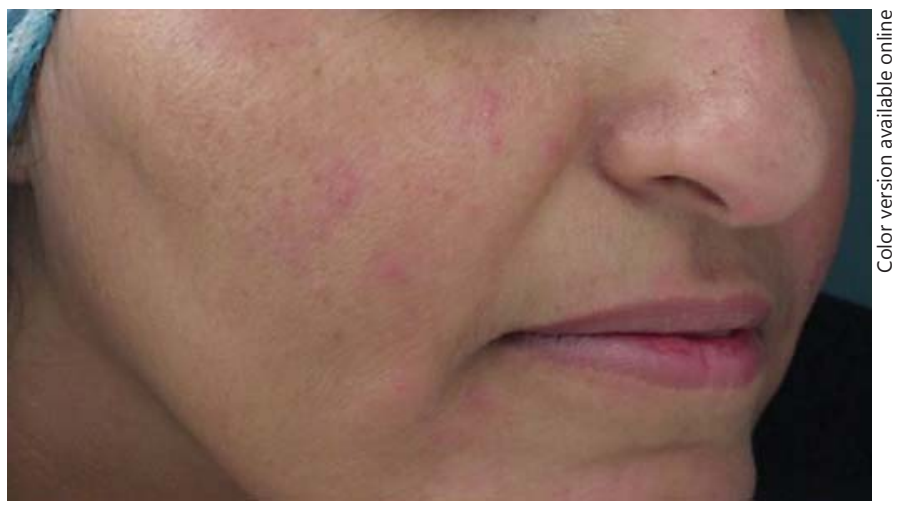

Fig. 1. 36-year-old patient, skin type 5, showing erythema over the malar area and glabella with papules and pustules.

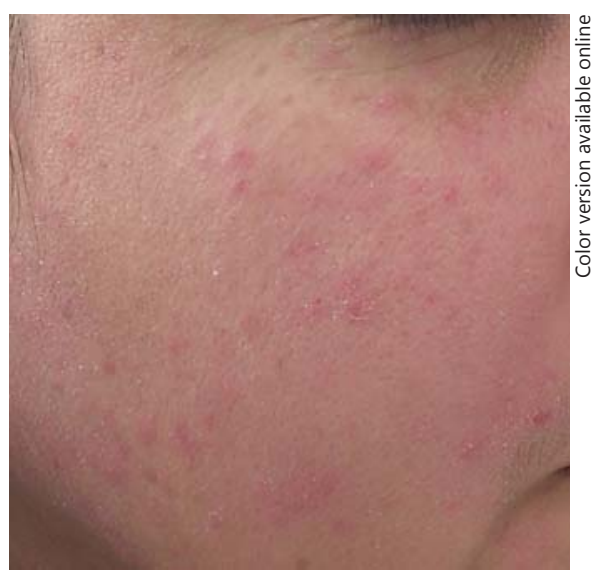

Fig. 2. 40-year-old patient, skin type 5, with papulopustules on erythematous background and scales.

(common in the healthy Swedish population) if it was not associated with flushing (which they called static telangiectasia).

Although the rosacea patients with and those without radiological evidence of CRS were clinically similar (midfacial erythema, telangiectasia with or without papules and pustules; fig. 1-3), ETR was more severe in patients with radiological evidence of CRS. The percentage of those with a family history in this study (10.7\%) was lower than that reported in the literature for light-skinned individuals (33\%) [1]. This could be explained by the different patient populations or by the known difficulty of appreciating erythema and telangiectasia in dark-skinned individuals [21].

As reported by many authors, skin barrier function is impaired in rosacea, proved by the reactive skin irritation 

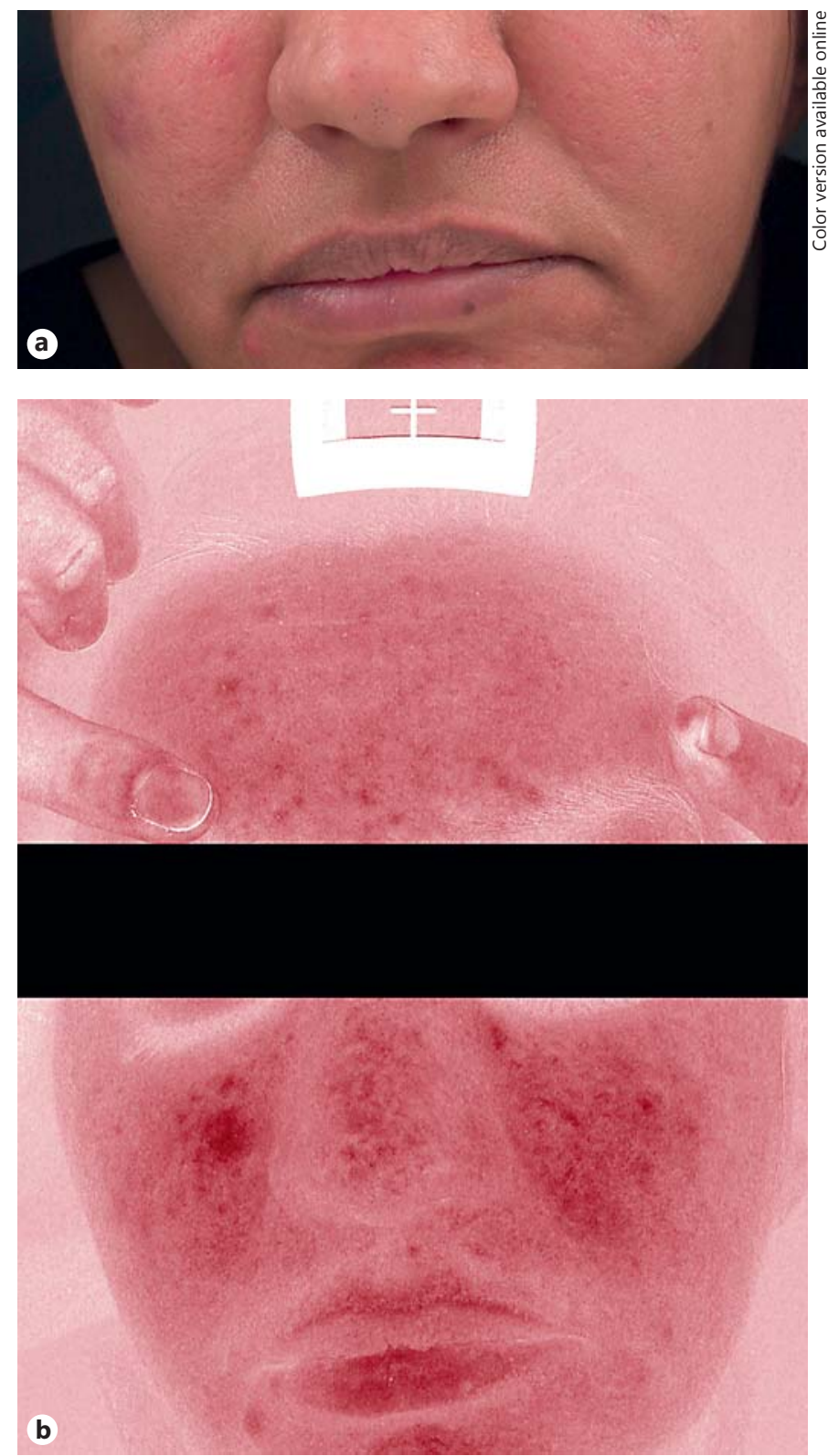

Fig. 3. a 46-year-old patient, skin type 6, with ETR and edematous plaque without VISIA Complexion Analysis. b VISIA Complexion Analysis image showing clear erythema and telangiectasia.

test in $100 \%$ of patients with ETR and $68 \%$ of patients with PPR [22, 23]. It is believed that skin irritation in rosacea could result in dermatitis that presents as scaling and dryness. This belief is supported by our findings of facial scaling, pruritus, and skin tightness, occurring in $100 \%$ of the patients (fig. 4).

Sun exposure was the most common aggravating factor for all patients, thereby indicating the inherent pho-
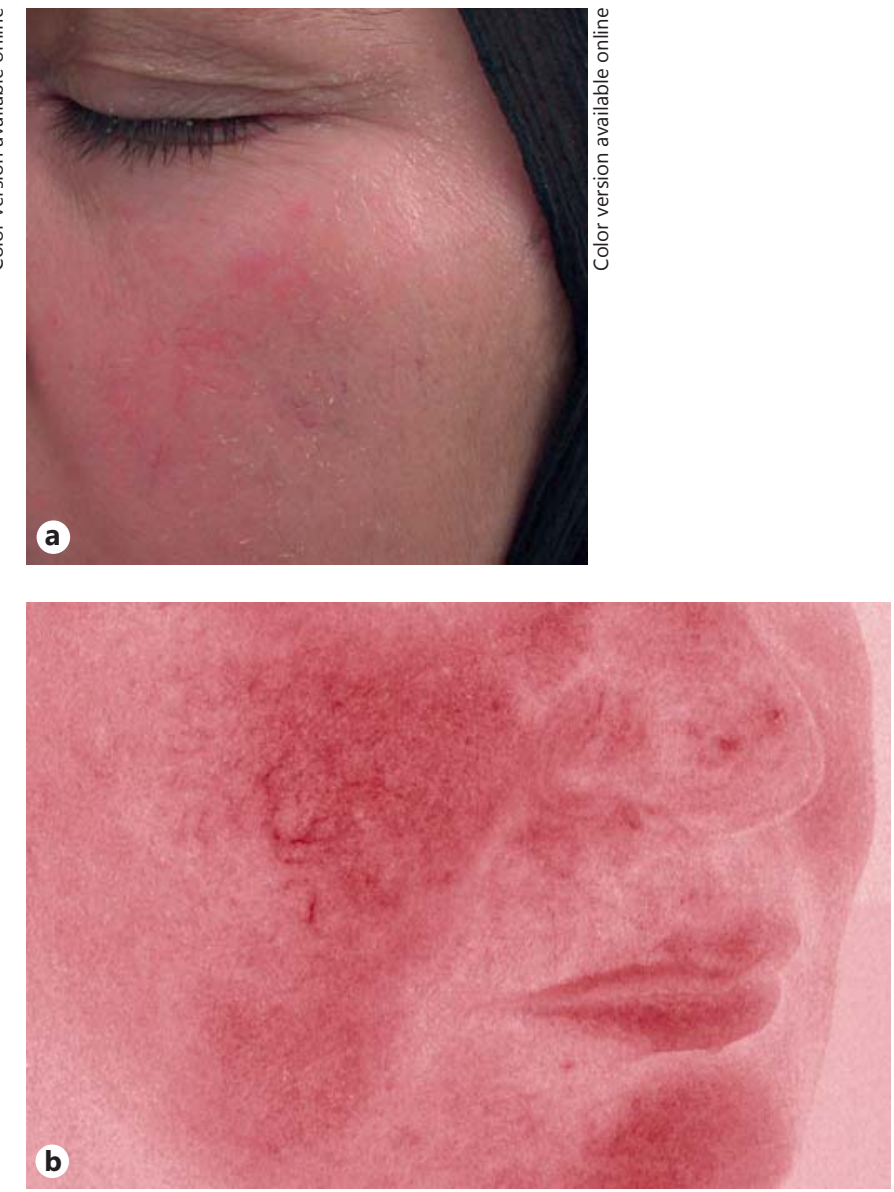

Fig. 4. a Rosacea with scales over the cheek and eyelid. b VISIA Complexion Analysis image showing erythema and telangiectasia.

tosensitive nature of rosacea that does not affect a dark skin complexion. Dust and sweat were significantly more often reported by the patients with radiological evidence of CRS; hence, both should be included as aggravating factors of rosacea with radiological evidence of CRS.

The percentage of patients with rosacea and radiological evidence of CRS who suffered from migraine (15.8\%) was lower than the percentages of rosacea patients with migraine ( 44 and $27 \%$ ) reported by Berg and Lidén [20] and Tan and Cunliffe [24]. The influence of racial differences might explain the lower incidence of migraine among our rosacea patients. In subjects $>40$ years old, Saberi et al. [25] reported a significant correlation between migraine and allergic rhinitis. It is sometimes difficult to differentiate between sinus headache and migraine, due to the frequent occurrence of nasal symptoms in migraine and the precipitation of migraine attacks by sinus inflammation $[25,26]$. This may 
explain the clustering of migraine sufferers among rosacea patients with CRS.

There are several limitations to this study. Unfortunately, it was underpowered due to the difficulties of recruiting patients and control groups for participation in the study and of performing paranasal X-rays rather than CT scanning to screen for CRS. However, the higher prevalence of radiological evidence of CRS in the study group supports the possibility that CRS and rosacea are associated diseases. It appears that there is a tissue interaction between the inflamed mucosal lining of the sinuses and the overlying skin in rosacea, mediated by cathelicidin and anatomical proximity.

\section{Conclusion}

Our study showed that rosacea was associated with CRS. The patients with this association showed severe and extensive vascular skin disease. This relationship could provide more therapeutic modalities in the future.

\section{Acknowledgments}

We would like to thank our statistician, all the residents who participated in data collection, and the secretaries who assisted in writing.

\section{Disclosure Statement}

The author has no conflicts of interest to disclose.

\section{References}

1 Bamford J: Rosacea: current thoughts on origin. Semin Cutan Med Surg 2001;20:199-206.

-2 Yamasaki K, Gallo RL: Rosacea as a disease of cathelicidins and skin innate immunity. J Investig Dermatol Symp Proc 2011;15:12-15.

3 Meylan E, Tschopp Jr, Karin M: Intracellular pattern recognition receptors in the host response. Nature 2006;442:39-44.

4 Takeda K, Kaisho T, Akira S: Toll-like receptors. Annu Rev Immunol 2003;21:335-376.

5 De Yang, Chen Q, Schmidt AP, et al: LL-37, the neutrophil granule- and epithelial cellderived cathelicidin, utilizes formyl peptide receptor-like 1 (FPRL1) as a receptor to chemoattract human peripheral blood neutrophils, monocytes, and T cells. J Exp Med 2000; 192:1069-1074.

6 Koczulla R, von Degenfeld G, Kupatt C, et al: An angiogenic role for the human peptide antibiotic LL-37/hCAP-18. J Clin Invest 2003; 111:1665-1172.

7 Yamasaki K, di Nardo A, Bardan A, et al: Increased serine protease activity and cathelicidin promotes skin inflammation in rosacea. Nat Med 2007;13:975-980.

8 Agerberth B, Charo J, Werr J, et al: The human antimicrobial and chemotactic peptide LL-37 and $\alpha$-defensins are expressed by specific lymphocyte and monocyte populations. Blood 2000;96:3086-3093.

9 Hase K, Murakami M, Iimura M, et al: Expression of LL-37 by human gastric epithelial cells as a potential host defense mechanism against Helicobacter pylori. Gastroenterology 2003; $125: 1613-1625$.
10 Stoeckelhuber M, Olzowy B, Ihler F, et al: Immunolocalization of antimicrobial and cytoskeletal components in the serous glands of human sinonasal mucosa. Histol Histopathol 2014, Epub ahead of print.

11 Ooi EH, Wormald PJ, Carney AS, et al: Human cathelicidin antimicrobial peptide is upregulated in the eosinophilic mucous subgroup of CRS patients. Am J Rhinol 2007;21 395-401.

12 Taub AF, Devita EC: Successful treatment of erythematotelangiectatic rosacea with pulsed light and radiofrequency. J Clin Aesthet Dermatol 2008;1:37-40.

13 El-Khalawany M, Mahmoud A, Mosbeh AS, et al: Role of Helicobacter pylori in common rosacea subtypes: a genotypic comparative study of Egyptian patients. J Dermatol 2012; 39:989-995.

14 Fitzpatrick TB, Ortonne J: Normal skin color and general consideration of pigmentary disorders; in Freedberg IM, Eisen AZ, Wolff K, et al (eds): Dermatology in General Medicine, ed 6. New York, McGraw-Hill, 2003, pp 820821.

15 Wilkin J, Dahl M, Detmar M, et al: Standard classification of rosacea: report of the National Rosacea Society expert committee on the classification and staging of rosacea. J Am Acad Dermatol 2002;46:584-587.

16 Mafee MF: Imaging of paranasal sinuses and rhinosinusitis. Clin Allergy Immunol 2007; 20:185-226.
17 Adil R, Qayyum A, Qayyum A: Correlation of $\mathrm{X}$-rays and computed tomography in paranasal sinus diseases. Pak Armed Forces Med J 2011;3:61-63.

18 Meltzer EO, Hamilos DL, Hadley JA, et al: Rhinosinusitis: establishing definitions for clinical research and patient care. Otolaryngol Head Neck Surg 2004;131(suppl):S1-S62.

19 Dirschka T, Tronnier H, Fölster-Holst R: Epithelial barrier function and atopic diathesis in rosacea and perioral dermatitis. Br J Dermatol 2004;150:1136-1141.

20 Berg M, Lidén S: An epidemiological study of rosacea. Acta Derm Venereol 1989;69:419423

21 Alexis AF: Rosacea in patients with skin of color: uncommon but not rare. Cutis 2010;86: 60-62.

22 Lonne-Rahm SB, Fischer T, Berg M: Stinging and rosacea. Acta Derm Venereol 1999;79: 460-461.

23 Corazza M, la Malfa W, Lombardi A, et al: Role of allergic contact dermatitis in rosacea. Contact Dermatitis 1997;37:40-41.

24 Tan SG, Cunliffe WJ: Rosacea and migraine. Br Med J 1976;1:21.

25 Saberi A, Nemati S, Shakib RJ, et al: Association between allergic rhinitis and migraine. J Res Med Sci 2012;17:508-512.

26 Cady RK, Schreiber CP: Sinus headache or migraine? Considerations in making a differential diagnosis. Neurology 2000;58(suppl 6): S10-S14. 